\title{
COLCHICINE REDUCES MYELIN THICKNESS AND AXOPLASM VOLUME
}

\author{
STEPHEN E. HUGHES, HARRY E. SLOAN, LEE B. JONES and BRUCE OAKLEY* \\ Division of Biological Sciences, University of Michigan, Ann Arbor, MI 48109 (U.S.A.)
}

(Received February 25th, 1983; Accepted March 15th, 1983)

Key words: colchicine - myelin - axoplasm - lingual nerve - chorda tympani netve - Schwann cell axonal transport - taste

A Silastic cuff containing either colchicine $(1 \% \mathrm{w} / \mathrm{v})$ or no colchicine was placed around the lingualchorda tympani nerve of the Mongolian gerbil. After 3 days of exposure to colchicine, the mean period of the myelin sheaths was $23 \%$ less than the period observed in nerves treated with cuffs lacking colchicine, while the average number of lamellae was unaltered. At the same time colchicine reduced the volume of axoplasm by an average of $19 \%$, an effect which was independent of fiber diameter.

As part of a series of investigations on the neurotrophic maintenance of taste buds and taste responses $[12,13,16]$, the present research was designed to evaluate the ultrastructural changes in the lingual-chorda tympani nerve caused by chronic exposure to colchicine. Earlier investigations had found that colchicine reduced the number of microtubules, and also increased the prominence of filaments in both neurons $[2,3,5,17]$ and glia $[4-6,11]$. In addition, colchicine is known to impair both fast $[2,8,15]$ and slow $[5,7]$ axonal transport. Here, we describe observations on colchicine-induced loss of axoplasm and thinning of myelin.

In order to correlate morphological changes in the nerve with physiological changes in taste activity, we evaluated the ultrastructural changes in axons exprsed to colchicine for 3 days, by which time colchicine had impaired axonal transport and substantially reduced chorda tympani taste responses [16]. A nerve cuff of Silastic (Medical Adhesive Silicon Type A, Dow Corning) was implanted around the lingual-chorda tympani nerve in each of 12 Mongolian gerbils (Meriones unguiculatus, 5-9 months old, 56-74 g body weight) by methods described elsewhere [16]. Six colchicine cuffs $(1 \% \mathrm{w} / \mathrm{v})$ and six control cuffs lacking colchicine were used. Three days after cuff implantation, the gerbils were anesthetized with ketamine $\mathrm{HCl}(330 \mathrm{mg} / \mathrm{kg}$ body weight i.m.) and sodium pentobarbital $(12.5 \mathrm{mg} / \mathrm{kg}$

- Author for correspondence at: Neuroscience Laboratory Building, University of Michigan, Ann Arbor, MI 48109, U.S.A. 
i.p.). The unbranched. $5.5 \mathrm{~mm}$ segment of the lingual-chorda tympani nerve. $2 \mathrm{~mm}$ proximal and $2 \mathrm{~mm}$ distal to the cuff. was removed along with the corresponding regment of the normal contralcteral nerve. The two segments were processed idenlically. Immediately after removal, the nerves were fixed for $\mathbf{2} \mathrm{h}$ in $2 \sigma_{0}$ glutaraldehyde in $0.2 \mathrm{M}$ sucrose buffered with $0.1 \mathrm{.1}$ cacodylate (pH 7.35), rinsed in the buffered sucrose, postixed for $2 \mathrm{~h}$ in buffered $2 \sigma_{0} \mathrm{OsO}_{4}$ and rinsed again. The nerver wete sained in $2 \omega^{\circ}$ aqueous uranyl acetate for $1 \mathrm{~h}$. dehydrated in ethanol. and embedded in Spurr's medium (Polysciences). Cross-sections were cut from the nerse segments just distal to the cuff and at a corresponding level in the contralateral netres. Sections were stained with lead eitrate and were examined in a Philip, F.M-3on dectron microsiope.

Analyis of dectron micrographs was aided by a digitizng graphics tablet and microcomputer (Apple (omputer. Inc.). All mearurements were made without knowledge of the nerte treatment. Myelin thickness (distance between inner and (nter lamellat) was determined by as craging measurements (typically 6 - 8 ) taken at 1 am inters als around the perimeter of each axon $>$ cross-sectional profile. The internal liber diameter was computed from measurements of the circtemference of the innermos myelin lamella. Aroplasm low was guantified by measuring the erossextional afcal enclosed by the asolemma and expressing this area as a percentage of the area collowed by the inncrmos mystin lamella.

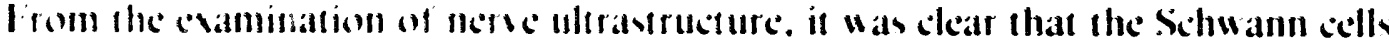

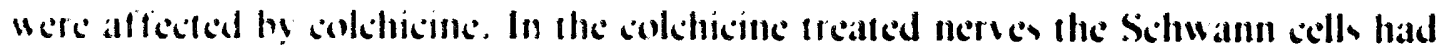

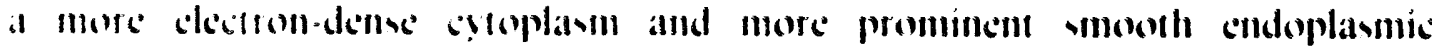
reliculum with sularged sisicrnate.

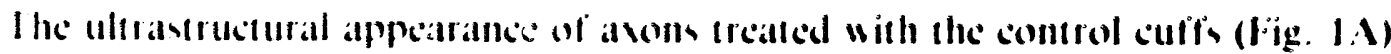
Wat similaf to the normal appeatance of the unt reatled entralateral nerves. In contrast. in colchicine-xposed mydinated axoms the number of microtubules 1 as substantially decreased, the neurofilaments were more prominent and often longitudinally disoriented, and small clusters of mitochondria were frequently iresent in the axoplasm. Colchicine also reduced the volume of axoplasm, which led to the retration of the atolemma away from the innermost lamella of the myelin (Fig. IB). As the volume of axoplasm decreased, it was not uncommon for the sheaths to collapse inward, producing folds in the myelin. In six colchicine-treated nerves there was a mean $190^{\circ}$ loss in axoplasm volume compared to six 3-day control cuffed nerves $(P<0.02, t$ test, two tailed). The actual reduction in colume was probably grealer than $190_{0}^{\circ}$ because the concurrent infolding of the myelin effectiveIy reduced the area enclosed by the myelin sheath. As calculated from the internal circumterence of the sheath, the internal fiber diameters in control-cuffed nerves $(2.43 \pm 1.03 \mu \mathrm{m}, \mathrm{M} \pm \mathrm{S} . \mathrm{D}$.) were not significantly different from those of fibers exposed to colchicine $(2.31 \pm 0.99 \mu \mathrm{m}, P>0.1)$, indicating that similar samples of avons were compared. We used internal, and not external, fiber diameters to avoid confounding measterements of axoplasm loss with the concurrent thinning of the 

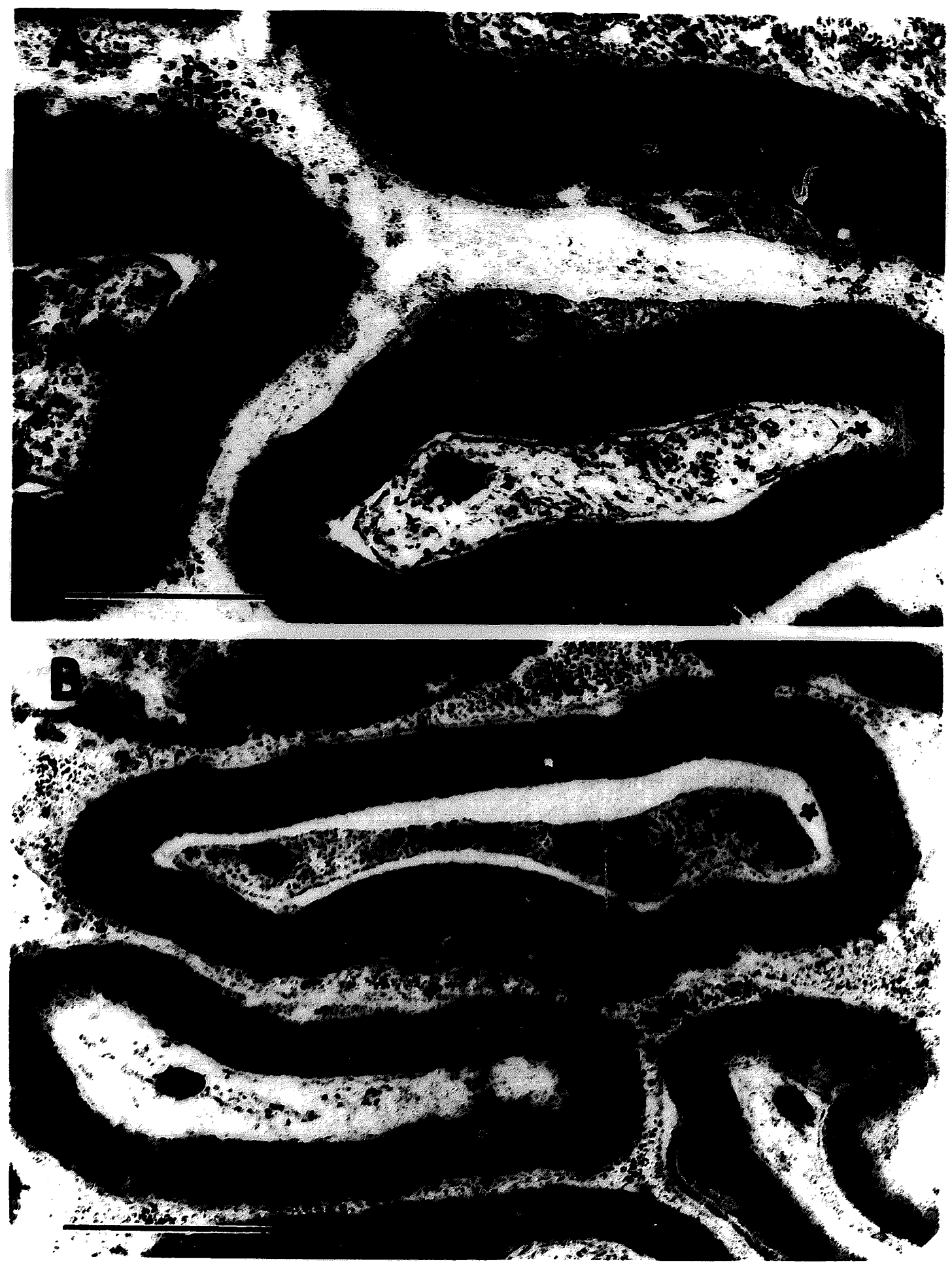

1.ig. 1. A: with a control Silastic cuff lacking colchicine the appearance of the axons after 3 days is similar to normal uncuffed nerves. Note the abundant microtubules. The shape of the axon profiles is representative of those present. B: exposure to a $1 \%$ colshicine cuff for 3 days reduced the number of microtubules and the thickness of the myelin. Adjacent axons showed differing degrees of axoplasm loss. The starred axon $(*)$ has 27 lamellae like the starred axon in $A$, but has thinner myelin. Bars $=1 \mu \mathrm{m}$. 


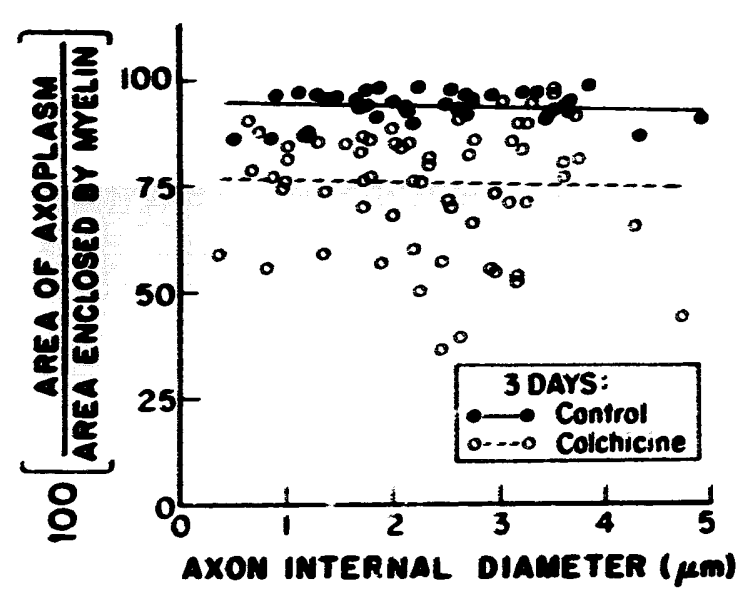

Fig. 2. Colchicine produced a significant reduction independent of fiber size in the percentage of axonal cross-sectional area occupied by axoplasm. In nerves treated with control cuffs, as in normal nerves, the axoplasm nearly filled the area surrounded by myelin. Lines are the least squares best fit. $n=68$ (colchicine) and 37 (control cuff).

myelin sheaths (see below). The near zero slope of the least squares best fit line in Fig. 2 (dashed line) indicates that the percentage of axoplasm volume lost was independent of fiber diameter, in contrast to an earlier report [5].

It is evident from the variability in Fig. 2 that axons reacted dissimilarly to the colchicine treatment. Axons with extensively reduced axoplasm were scattered throughout the nerve. When both the exposure to colchicine and fiber diameter were controlled by comparing adjacent fibers of similar diameter, substantial differences in axoplasm loss were still frequently or:served (cf. Fig. 1B). It is reasonable to conclude that the variation among axons in axoplasm loss was primarily determined by differential susceptibility of axons to colchicine rather than by differences in diameter or colchicine exposure.

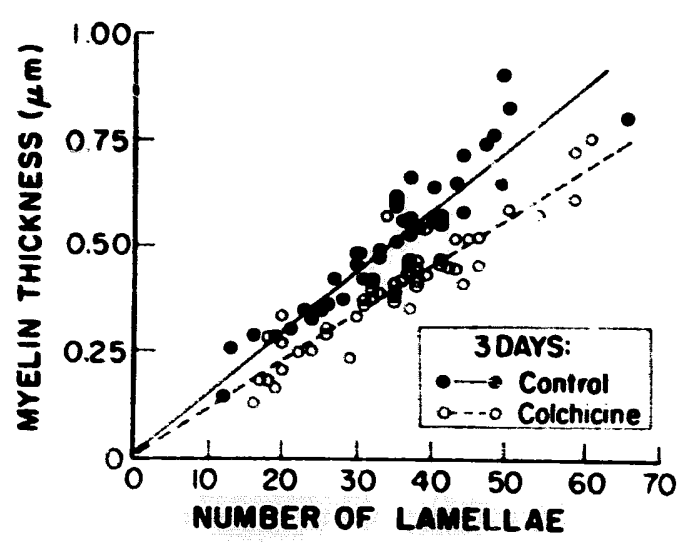

Fig. 3. For all fiber diameters the thickness of the myelin sheath was reduced by 3 days of exposure to colchicine. The slope of the line gives the period for both control and colchicine-treated groups. Lines are the least squares best fit. $n=50$ (colchicine) and 46 (control cuff). 
The ability of colchicine to impair axonal transport has been well documented [2, $8,15]$ and was verified in the present research by observing impaired cholinesterase transport [16]. The accumulation of mitochondria provided additional evidence for impaired axonal transport. We hypothesize that the colchicine-containing cuff reduced axonal transport, which led to a local depletion of axoplasm.

Myelin thickness and the number of lamellae were measured in axons from six nerves treated with colchicine-cuffs and six nerves treated with control cuffs. Colchicine reduced the mean thickness of the myelin sheath to $0.40 \pm 0.13 \mu \mathrm{m}$ from the normal values found with control cuff treatment $(0.51 \pm 0.16 \mu \mathrm{m})$. As an example, the two starred axons (*) in Fig. 1 each have 27 lamellae, yet the myelin sheath of the colchicine treated axon is $32 \%$ thinner. The colchici.e-induced thinning of the myelin sheaths was directly proportional to the number of lamellae. The Pearson correlation coefficients are $r: \cdots+0.92$ (control cuff) and $r=+0.93$ (colchicinecontaining cuff) for the least squares best fit lines in Fig. 3.

Tite myelin sheath's period (distance between the centers of the major dense lines) was calculated in individual axons by dividing the myelin thickness by the number of lamellae. The mean period in axons with control cuffs was $14.7 \pm 1.8 \mathrm{~nm}$, which is similar to other electron microscopic measurements of myelin period in mammalian peripheral nerves $[10,14]$. Three days of colchicine treatment decreased the mean period by $23 \%$ to $11.3 \pm 1.7 \mathrm{~nm}$. The reduced myelin period is graphically displayed by the slopes of the lines in Fig. $3(P<0.01, t$ test). The thinning of the myelin was not the result of lost lamellae; colchicine treated axons had a mean of $35.2 \pm 11.0$ lamellae compared to $34.7 \pm 10.5$ lamellae for axons of nerves with control cuffs. Nor was the shrinkage confined to either the inner or outer lamellae; at $150,000 \mathrm{X}$ the reduction in period appear to be even across the width of the sheath.

The breakdown of myelin caused by other toxic chemicals generally results from direct attack on the myelin or from breakdown secondary to the destruction of the glial cells [9]. In both cases myelin breakdown is characterized by the formation of vacuoles at the intraperiod lines [1, 18]. However, colchicine treatment for 3 days was not associated with vacuole formation. Nonetheless, these more subtle effects of colchicine upon myelin may have stemmed from changes in the Schwann cells [9].

In summary, the chronic application of a colchicine-containing Silastic cuff to the lingual-chorda tympani nerve produced a partial loss of axoplasm and thinner myelin lamellae. Specifically, three days of colchicine treatment produced a mean $19 \%$ loss of axoplasm volume in both large and small myelinated fibers and a $23 \%$ reduction in the myelin period.

We would like to thank Mr. Steven Scherer for helpful comments on the manuscript. This work was supported by NIH Grant NS-07072.

I Blakemore, W.F., Invasion of Schwann cells into the spinal cord of the rat following local injections of lysolecithin, Neuropathol. Appl. Neurobiol., 2 (1976) 21-39. 
2 Fink. B.R., Byers, M.R. and Middaugh, M.E., Dynamics of colchicine efrects on rapid transport and axonal morphclogy, Brain Res., 56 (1973) 299-311.

3 Freide, R.L. and Ho, K.-C., The relation of axonal transport of mitochondria with microtubules and other axoplasmic organelles, J. Physiol. (Lond.), 265 (1977) 507-519.

4 Hansson, H.-A. and Norström, A., Glial reactions induced by colchicine-treatment of the hypothalamic-neurohypophyseal system, Z. Zellforsch., 113 (1971) 294-310.

5 Hansson, H.-A. and Sjëstrand, J. Ultrastructural effects of colchicine on the hypoglossal and dorsal vagal neurons of the rabbit, Brain Res., 35 (1971) 379-396.

6 Jacobs, J.M., Cavenagh, J.B. and Chen, F.C.-K., Spinal subarachnoid injection of colchicine in rats, J. neurol. Sci., 17 (1972) $461-4 * 0$.

7 Karlsson. J.-O. Hansson, H.-A. and Sjöstrand, J., Effect of colchicine on axonal transport and morphology of retinal ganglion silis. 7 . Zellforsch., 115 (1971) 265-283.

8 Kreutzberg. G.W.. Neuronal dynamics and axonal flow. IV. Blockade of intra-axonal enzyme transport by colchicine, Proc. nat. Acad. Sci. U.S.A. 62 (1969) 722-728.

9 Ludwin, S.K.. Pathology of demyelination and remyelination. In S.G. Waxman and J.M. Ritchie (Eds.) Demyelinating Disease: Basic and Clinical Electrophysiology, Raven Press, New York, 1981. pp. $123-168$.

10 Napolitano. L.M. and Scallen. T.J.. Otservations on the fine siructure of peripheral nerve myelin, Anat. Rec., 163 (1969) 1-6.

11 Norstrom. A., Hansson. H.-A. and Sjostrand, J., Effects of colchicine on axonal transport and ultrastructure of the hypothalamo-neurohypophyseal system of the rat, Z. Z:llforsch., 113 (1971) $271-293$.

12 Oakley. B.. Chu, J.5. and Jones, L.B., Axonal transport maintains taste responses, Brain Res.. 221 (1981) 289-298.

13 Oakley. B., Jones, L.B, and Hosley, M.A., The effect of nerve stump length upon mammalian taste responses, Brain Res.. 194 (1980) 213-218.

it Sjostrand. F.S.. The lamellated structure of the nerve myelin sheath as revealed by high resolution electron microscopy, Evperiemtia. 9 (1953) 68 . 69.

15 Sjostrand. J., Frizell. M. and Hasselgren, P.-O., Effects of eolchicinc on asonal transport in peripheral nerves. I. Neurochem., 17 (1970) $1563-1570$.

16 Sloan, H.E.., Hughes, S.E. and Oakley. B., Chronic impairment of axcral transport climinates taste responses and taste buds. J. Neurosci., 3 (1983) $117-123$.

17 Wisniewski, H., Shelanski, M.L. and Terry. R.D., Effects of mito ic spindle inhibitors on neurotubules and neurofilaments in anterior horn cells, J. Cell Biol., 38 (1968) 224-229.

18 Yajima, K. and Suzuki, K., Uitrastructural changes of oligodendroglial and myelin sheaths induced by ethidium bromide. Neuropathol. Appl. Neurobiol., 5 (1979) 49-62. 